\title{
Super-knock Suppression Using Split Injection in a Turbo-Charged GDI Engine
}

\author{
LV Meng $^{1}$, LI Yun ${ }^{1,2}$, HU Xiaoming ${ }^{1}$, LIU Yongchen ${ }^{1}$, CHANG Lv ${ }^{1}$ \\ ${ }^{1}$ Huaiyin Institute of Technology, China \\ ${ }^{2}$ Key Laboratory for Traffic and Transportation Security of Jiangsu Province, USA
}

\begin{abstract}
Super-knock, which occurs under low-speed and big load operate condition in turbo-charged gasoline direct injection (TGDI) engine, is an abnormal combustion phenomenon. Due to its potential to cause degradation of the engine, super knock has become the main obstacle for increasing specific power and lowing fuel consumption of engine. In order to investigate suppress strategies of super-knock, split injection is applied on a high boosted GDI engine. The results shows that: super knock correlates to some extent with split injection; further study shows that appropriate split injection duty ratio and the end of second injection time could reduce super knock effectively.
\end{abstract}

\section{Introduction}

With the energy crisis and environment pollution spreading all over the world, the technology that gasoline direct injection combine with turbo charger, as an important measure to reduce fuel consumption and improve emission of engine, is developed rapidly. An abnormal combustion phenomenon has been found in highly boosted SI engine, especially in turbo-charged gasoline direct injection (TGDI) engine, which is termed as super knock (Volkswagen calls it Super Knock [1], AVL names it Mega-Knock[2] and Pre-Ignition in IFP[3]),. Super knock is characterized as a pre-ignition usually followed by heavy detonation and sharp metal knock voice. Super knock occurs randomly and in an uncontrolled style, often just single occurs but it occasionally takes place two or more times in sequence and then disappears spontaneously, it is difficult to identify the root cause for this phenomenon and to develop solutions to eliminate it. The occurrence of super knock usually accompany with high frequency pressure wave, the maximum peak fire pressure in cylinder may reach up to 350 Bar which bring fatal damage to engine, Although it is neither possible nor practical to eliminate super knock; thus, a suitable way for mitigating super is to weaken the conditions favor super knock .

In order to suppress super knock, many studies have been done globally, such as spark plug gap, heat value, negative electrode has little effect on super knock is proved by Takuya Inoue from NGK[4]. Volkswagen Company and Vienna University [1] decreases super knock frequency by optimizing the structure of combustion chamber, eventually enhance brake mean effective pressure(BMEP). Christoph Dahnz[5] from KIT University proves that hot spot, which is widely believed as the cause for super knock, locating in combustion chamber randomly not the particular place, on the other hand, points out super knock maybe result in the interaction between fuel and oil droplets from wall by fuel inject splash. Southwest Research Institute proposes that mechanism of super knock maybe resulted from HC which exist in the crevice of combustion chamber. Akram Zahdeh[6] reduces super knock frequency substantially using split injection on a TGDI engine. In this paper, split injection strategy will be carried on a TGDI engine to restrain super knock, especially the end of second injection time (EOI) and the ratio of second injection (ROI) will be studied carefully.

\section{Experimental setup and method}

\subsection{Test Engine}

The test engine under study is a turbo-charged gasoline direct injection (TGDI) engine with 4-cylinder in line, turbo-charged and inter-cooling, dual-over head camshaft with central spark plug and lateral injector configuration. The basic engine parameters are presented in Table1 unless otherwise noted. The engine is equipped with a high-pressure fuel pump whose maximum supply fuel pressure is $20 \mathrm{MPa}$.

Table 1. Test Engine Parameters.

\begin{tabular}{ll}
\hline Project & Parameter \\
\hline Engine Type & 4 Cylinders in Line \\
Stroke/mm & 86 \\
Bore $/ \mathrm{mm}$ & 86 \\
Displacement $/ \mathrm{L}$ & 1998 \\
Compress Ratio & 9.75
\end{tabular}


Fuel Charged Mid-Cooling

RON93

Double Parallel Turbo Water Cooling Bench

\subsection{Test Bench}

The test is carried in the laboratory which is equipped with air condition system. The test bench is equipped with coolant temperature control system, fuel temperature control system and AVL electric dynamometer. The engine is equipped with the standard temperature and pressure sensors in the inlet and outlet systems, lubricating oil and coolant circuit, etc. Further measurement systems included wide band O2-sensor, fuel consumption meter and smoker meter. Cylinder pressure transducers in each of the four cylinders were employed to measure cylinder pressure with high temporal resolution and the transducer type is $6025 \mathrm{C}$ by Kistler. The equipments are used in test as shown in Table 2. The temperature in test room is controlled around $25 \pm 1{ }^{\circ} \mathrm{C}$, coolant temperature around $88 \pm 3{ }^{\circ} \mathrm{C}$ and lubricating oil temperature around $95 \pm 3^{\circ} \mathrm{C}$. In test, INCA5.4 software which through ETAS ES590 connects ECU is used to control ECU functions and engine parameters.

Table 2. Test Equipment.

\begin{tabular}{llc}
\hline Equipment & Type & Manufacturer \\
\hline Electric Dynamometer & INDY S12-2 & AVL \\
Fuel Consumption Meter & KMA4000 & AVL \\
LAMBDA Instrument & LA4 & ETAS \\
Coolant system & 553-200L & AVL \\
\hline
\end{tabular}

\section{Experimental methods}

\section{1 super knock test}

Accompany with sharply metal knock voice and high frequency shock wave, Super knock randomly occurs before igniting. Kinds of methods are used to identify super knock, such as voice, visual, peak fire pressure, knock intensity and ion current etc. So far, there is no method can be confirmed as a criterion for identify super knock. In this study, peak fire pressure and abnormal voice will be used to identify super knock.

\subsection{Experimental progress and method}

Electric dynamometer is used to motor engine to start and then warm up till temperature of coolant and oil reach up to the target value, then begin super knock test. Identify super knock by combustion analysis meter connect cylinder pressure sensor. Due to its random occurrence and potential damage to engine, every case will continue for an hour, and record the super knock frequency. Stop test when super knock times above 10 in order to protect engine and sensor from destroy.
Because super knock occurs at low-speed and big load condition, the test is carried at $2000 \mathrm{rpm}$. Test Conditions of engine as followed by Table 3 . In the experiment, ROI and EOI is controlled by INCA5.4 independently and cylinder pressure data is recorded by the AVL Indicom system.

Table 3. Engine Super Knock Test Conditions.

\begin{tabular}{ll}
\hline Type & parameter \\
\hline Speed/rpm & 2000 \\
Fuel Rail Pressure/Mpa & 18 \\
Inlet Pressure/MPa & 0.19 \\
Brake Mean Effective Pressure & 2.0 \\
/MPa & \\
Start of Injection(SOI) $/{ }^{\circ} \mathrm{CA}$ & 300 \\
BTDC) & \\
Injection Pulse Width $/ \mathrm{ms}$ & 6.2 \\
Lambda & 0.88 \\
\hline
\end{tabular}

\section{Result and discuss}

Super knock randomly occurs and disappears spontaneously; the maximum peak fire pressure is 2 times than common combustion. Figure1 compares cylinder pressure traces for normal combustion and abnormal combustion with super knock, as can be clearly seen in Figure 1, both 59 and 62 cycles is typical super knock phenomenon while others is normal combustion traces.

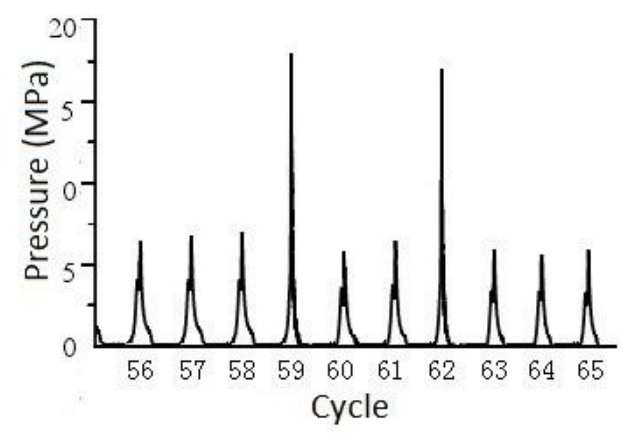

Figure1. Occurrence of Super Knock.

\subsection{Split Injection}

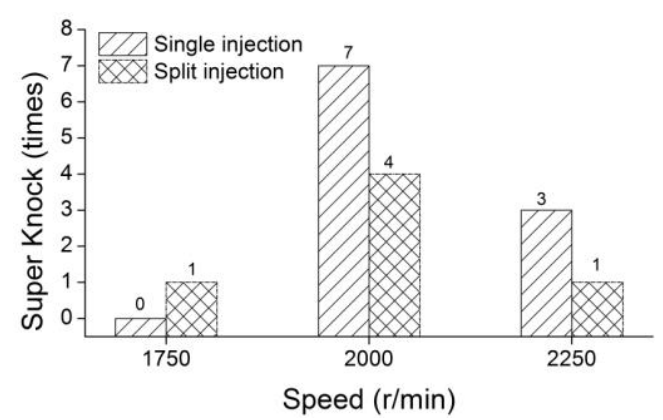

Figure 2. Super Knock Times for Single/Split Injection.

Figure 2 compares super knock times for single injection with split injection under low-speed and full-load 
condition. Obviously, As can be seen in figure 3.2, when split injection is applied to engine, super knock times is more than single one, proves that split injection could suppress super knock.

\subsection{EOl}

Figure 3 compares super knocks with different end of injection time (EOI), while SOI time and EOI duty ratio and injection pulse width is constant, adjusting EOI time from BTDC180 ${ }^{\circ} \mathrm{CA}$ to BTDC120 ${ }^{\circ} \mathrm{CA}$. The result can be in Figure 3, super knock occurs only at the BTDC $180^{\circ} \mathrm{CA}$ condition. This result can be reason for that, the more EOI advanced, the more homogeneous the mixture become, which benefit for flame spread;

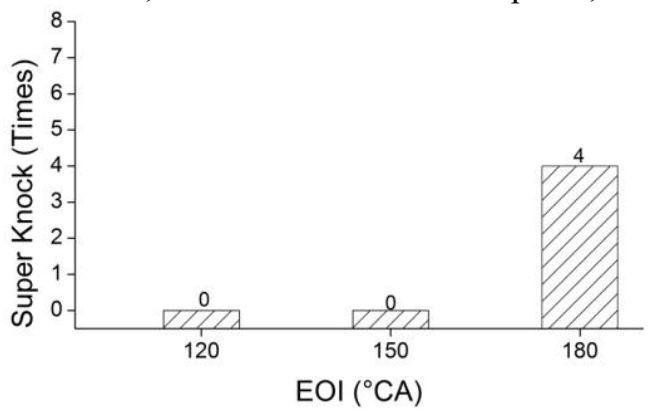

Figure 3. Super Knock Times for Different EOI.

\section{$4.3 \mathrm{ROI}$}

In order to study the influence of the ratio of second injection (ROI) on super knock, adjusting ROI while SOI time and EOI time and injection pulse width is fixed in test. The super knock frequency is shown in Figure 4. As shown that, with increasing of ROI, super knock frequency decreases gradually. The phenomenon is because combustion chamber temperature lower with increasing of EOI fuel quantity; On the other hand, when EOI fuel quantity is increased, both of emission smoke intensity[7] and lubricant oil dilution is risen up[8].

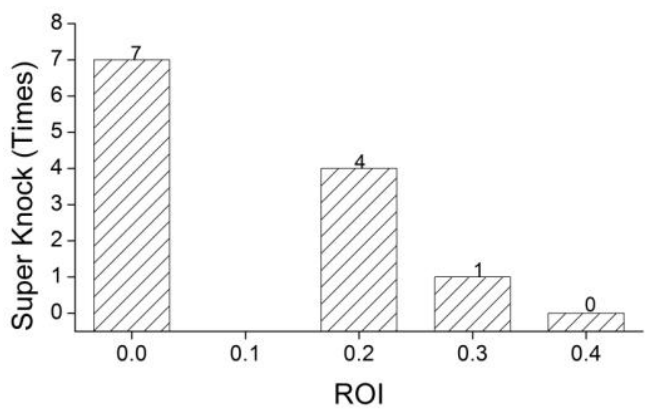

Figure 4. Super Knock Frequency for Different ROI.

\section{Conclusion}

On a highly boosted GDI engine, the impact of Split injection strategies on super knock is studied, especially end of second injection time(EOI) and ratio of second injection(ROI), the result shows that:

1) Using split injection strategy, the frequency of super knock is lower than single injection strategy.

2) Super knock frequency would be decreased when Increasing ROI ;

3) Modifying EOI benefit for restraining super knock.

\section{References}

1. Jurgen W, Marc D, Emanula M, Limits on Downsizing in Spark Ignition Engines due to Preignition[J]. MTZ, 2009, 70(5):56-61.

2. Winklhofer E, Hirsch A, Kapus P, et al. TC GDI engines at very high power density-irregular combustion and thermal risk [C]. SAE Paper 200924-0056.

3. Jean-Marc Zaccardi, Laurent Duval and Alexandre Pagot. Development of Specific Tools forAnalysis and Quantification of Pre-ignition in a Boosted SI Engine[C]. SAE Paper 2009-01-1795.

4. Inoue $\mathrm{T}$, Inoue $\mathrm{Y}$, Ishikawa $\mathrm{M}$. Abnormal combustion in a highly boosted SI engine - the occurrence of super knock[C]. SAE Paper 2012-011141.

5. Christoph D, kyung M, Ulrich S, et al. Investigations on Pre-Ignition in Highly Supercharged SI Engines[C] SAE Paper 2010-01-0355

6. Zahdeh A, Rothenberger P, Nguyen W, et al. Fundamental approach to investigate pre- ignition in boosted SI engines [J]. SAE International Journal of Engines, 2011, 4(1): 246-273.

7. AkramZ,Peter R, Wai N, et al. Fundamental Approach to Investigate Pre-Ignition in Boosted SI Engines [C]. SAE Paper 2011-01-0340

8. Lei Xiao-hu, Wang Yan-jun, Wang Jian-xin, et al. The Principal and Practice of Combustion Controlling in an In-cylinder Gasoline Direct Injection Engine. Chinese Internal combustion engine engineering, 2004, 25(3):4-7. 